\title{
Successes and Challenges of Laser-Induced Breakdown Spectroscopy (LIBS) Applied to Chemical Analyses of Geological Samples.
}

\author{
M. Darby Dyar ${ }^{1}$, Elly A. Breves ${ }^{1}$, Thomas F. Boucher ${ }^{2}$, and Sridhar Mahadevan ${ }^{2}$. \\ 1. Department of Astronomy, Mount Holyoke College, South Hadley, MA 01075 USA. \\ 2. School of Computer Science, University of Massachusetts, Amherst, MA, 01003 USA.
}

The laser-induced breakdown spectrometer (LIBS) on the ChemCam instrument [1] on the Curiosity rover on Mars has played a vital role in bringing this new technology to the attention of the scientific community. With over 100,000 spectra from individual laser shots collected to date and many more in progress, ChemCam has amply demonstrated the robustness of this technique for remote applications in difficult environments. However, the $\pm 10-20 \%$ accuracy for major and minor elements analyzed by the ChemCam LIBS [2] currently limits its usefulness to semi-quantitative analyses; comparable issues also limit other geological applications of LIBS in scenarios with geologically diverse samples. This paper discusses successes and challenges being addressed in improving LIBS accuracies and bringing this technique to its full potential in applications across the geological community.

ChemCam likely represents LIBS' greatest success to date. It can analyze a $\sim 400 \mu \mathrm{m}$ spot from a distance of up to $7 \mathrm{~m}$ [1], while other LIBS applications are probing even smaller spots at close range [3] and larger targets at distances beyond $100 \mathrm{~m}$ [4]. LIBS instruments shine in situations where standoff capability is desirable, such as field work in inaccessible locations (ranging from the deep seas on Earth to asteroids and other planets) and in hazardous locales (e.g., nuclear storage facilities). The laser can also ablate through coatings, creating depth profiles of elemental concentrations [5]. Elements from $\mathrm{H}$ to $\mathrm{Pb}$ are analyzed simultaneously from each spectrum. The combination of speed, versatility, standoff capability, and depth profiling has allowed ChemCam to contribute to interpretations of Mars geology made by the Mars Science Lab mission, and many further applications on Earth and elsewhere in our solar system are already in use or are planned.

However, there remain many challenges to improving LIBS accuracy in chemical analyses of geological samples. Primary among them is the fact that interactions in a LIBS plasma are too complex to be modeled theoretically, requiring the use of empirical calibrations. The intensity of any given LIBS emission line is not directly proportional to the concentration of the emitting atom due to matrix effects arising from collisional interactions within the plasma, laser-to-sample coupling efficiency, and selfabsorption. The local atmosphere and its breakdown products also interact with ablated surface material in the plasma, and pressure influences plasma evolution. Finally, there are distinct transition probabilities associated with each element and each emission line. Geological materials have multielement, highly variable compositions and thus create very complex plasmas. For all of these reasons, conventional univariate techniques, which depend on classical calibration curves using the relationship between peak area and concentration in standards, are not useful in geological samples [6,7]. To address these challenges, larger spectral libraries acquired under varying distances, ablation/collection angles, and environmental conditions are needed, along with sophisticated multivariate analysis models that can produce truly quantiative chemical analyses. Work is in progress to address all these issues, as follows.

1. The effect of distance on LIBS spectra from ChemCam is being studied [6] using the globallyhomogeneous composition of dust to derive a distance correction based on ChemCam data acquired at varying distances. This correction, which is currently calibrated using individual emission lines, 
minimizes change in compositional predictions for the same target measured at different distances. Work is in progress using binary classifiers to expand the method to all pixels in the Mars data.

2. Initial experiments with geometry suggest that significant variations in signal can occur as a result of varying ablation and collection angles, and that the density and refractive properties of the samples being studied are also important. It is as yet unclear if this effect is related to the volume of material being ablated (a function of many variables including coupling, composition, and density) or the refractive properties of the sample (that vary with density and surface texture/roughness). Other experimental variables that we are addressing include shot-to-shot variability, energy density, and coupling efficiency.

3. Our group has assembled a suite of $>3,000$ geological samples from a wide variety of geological parageneses for which complete or nearly complete chemical analyses are available. Acquisition of LIBS spectra of all these samples using 50 shots at each of five locations on pressed powder pellets is in progress under air (Earth), vacuum (Moon and asteroids), and Mars (low $\mathrm{P}, \mathrm{CO}_{2}$ ) surface conditions. These data are being used to develop multivariate models to predict elemental composition of major, minor, and even trace elements.

4. Differences among spectra acquired using dissimilar instruments or calibration sets remain even when all experimental variables have been accounted for. These must be addressed using sophisticated statistical techniques that can combine information about the instruments and the LIBS elemental analysis. This task is ideally suited to the field of transfer learning, where we are addressing differences among instruments using manifold regression [7] to project the different instruments' data onto a shared latent manifold of the same dimensionality. To date, we have compared the performance of ten different models, and observed that a novel variant of locally-linear embedding regression provides the most accurate predictions of chemical composition on a small, 100-sample dataset.

5. Depending on the ratio between LIBS beam size and the grain size of geological materials being probed, LIBS may or may not produce a representative bulk rock analysis or an individual mineral analysis. Models to guide sampling design have been developed [8]. We are exploring techniques to identify minerals by stoichiometry or through use of elemental ratios that show great promise.

Laser-induced breakdown spectroscopy presents a tremendous opportunity for development of novel chemical analysis techniques for in situ or stand-off geological applications, and the technique shows great promise. Challenges confronting quantitative analysis capabilities are recognized and strategies to address them have been formulated and are currently being carried out. LIBS has a bright future in geological applications.

[1] R Wiens et al., Space Science Reviews (2012) DOI 10.1007/s11214-012-9902-4.

[2] R Wiens et al., Spectrochimica Acta 82 (2013) p. 1-27.

[3] C McManus et al., Applied Optics 47 (2008) p. G72-G79.

[4] K Stelmaszczyk et al., Applied Physics Letters 85 (2004) p. 3977-3979.

[5] H Newsom et al., Lunar and Planetary Science Conference (2012) Abstract 2477.

[6] A Mezzacappa et al., Lunar and Planetary Science Conference (2014) Abstract 1517.

[7] D Pardoe and P Stone, Proceedings 27th International Conference on Machine Learning (2007).

[8] M McCanta et al., Planetary and Space Science 81 (2013) p. 48-54.

[9] The authors acknowledge funding from NSF grant CHE-1306133 and NASA grants NNX09AL21G and NNX12AK84G. 